\title{
BMJ Open Maternal mental health and infant and young child undernutrition: protocol for a systematic review
}

Manisha Singh (D) , ${ }^{1}$ Tomasina Stacey (D) , ${ }^{2}$ Julie Abayomi, ${ }^{3}$ Padam Simkhada ${ }^{1}$

To cite: Singh M, Stacey T, Abayomi J, et al. Maternal mental health and infant and young child undernutrition: protocol for a systematic review. BMJ Open 2021;11:e044989. doi:10.1136/ bmjopen-2020-044989

- Prepublication history and additional supplemental material for this paper are available online. To view these files, please visit the journal online (http://dx.doi.org/10.1136/ bmjopen-2020-044989).

Received 21 September 2020 Accepted 27 July 2021

Check for updates

(c) Author(s) (or their employer(s)) 2021. Re-use permitted under CC BY-NC. No commercial re-use. See rights and permissions. Published by BMJ.

${ }^{1}$ School of Human and Health Sciences, University of Huddersfield, Huddersfield, UK ${ }^{2}$ Department of Nursing and Midwifery, University of Huddersfield, Huddersfield, UK

${ }^{3}$ Department of Allied Health and Social Care, Edge Hill University, Ormskirk, UK

Correspondence to

Manisha Singh;

manisha.singh@hud.ac.uk

\section{ABSTRACT}

Introduction Mental health disorder, particularly depression, is one of the leading causes of 'disease related disability' in women that both affects the women but has adverse effect on their children. This can have an impact on mothers' capacity of child care which ultimately increases the risk of infection, malnutrition, impaired growth and behavioural problems in children that might extend to adulthood too. Diminished child growth has an irreversible effect both short and long terms, affecting physical growth, brain development, performance in education, working capacity and increased risks to noncommunicable diseases. To date, the reviews conducted are only limited to few countries or maternal depression or certain age group of children. Our aim is to provide a global perspective focusing on all early childhood undernutrition (under 5 years) and to see if the association between maternal mental health and child undernutrition has yielded similar or different result. Furthermore, we intend to explore the risk factors associated with copresence of maternal mental health issues and undernutrition in children.

Methods and analysis MEDLINE (PubMed), PsycINF0, CINAHL, Cochrane Library, Global Health Library Relevant reports from the WHO, United Nations of Children Education Fund and organisations working in maternal and child health will also be searched. Database of systematic reviews and database of abstracts of reviews of effects will also be searched for relevant literature. Papers published from 1995 to 2020 in English will be included. Title, abstract or both will be screened independently by reviewers. For data analysis and synthesis, we will present all the outcomes mentioned in the studies and a subgroup analysis for age and sex will be conducted. This study aims to conduct a meta-analysis.

Ethics and dissemination Ethical approval is not required to conduct this review. PROSPERO registration number CRD42020189315.

\section{INTRODUCTION}

Malnutrition is the deficiency or excess of nutrient intake that imbalances nutrients or causes impairment of utilisation of nutrients. ${ }^{1}$ It is broadly classified into three major categories "undernutrition", "overweight, obesity and diet related Non- communicable disease" and "micronutrient related malnutrition". This study will focus on both acute and

\section{Strengths and limitations of this study}

This systematic review appears to be the first to provide a global evidence of association between maternal mental health and undernutrition in children under 5 years of age.

- This review will be the first to document prevalence and if possible, different risk factors associated with copresence of maternal mental health and undernutrition in children globally.

- Cross-cultural validity of different data collection tools will be considered in the study.

- Studies published from 1995 to 2020 are only included in the review. Any studies published prior to 1995 are excluded.

chronic form of undernutrition in children under 5 years of age.

Undernutrition is a global public health problem where combating it has become one of the major priorities and challenge around the world. One or other form of malnutrition affects every nation. ${ }^{2}$ Recent studies show that 45 million children under 5 are wasted (low weight for height), and 149 million stunted, with highest prevalence reported in low-income and middle-income countries (LMICs). ${ }^{12}$ Furthermore, $45 \%$ of annual child mortality in under 5 children is linked to undernutrition in LMICs. $^{2}$ A significant achievement has been made in combating malnutrition, the mortality of children aged under 5 has dropped from 12 million in 1990 to 5.2 million in 2019 , one of the reasons being the significant achievements made in combating malnutrition. ${ }^{3}$ However, the Global Nutrition report, 2017, shows that the progress achieved to date, is not quick enough to meet internationally agreed nutritional goals as Sustainable Development Goals (SDG). ${ }^{4}$

Undernutrition does not just impair the physical growth, it also increases the risk of morbidity and mortality and affects mental and physical capability along with fertility. ${ }^{5}$ 
An extensive amount of research has been done to understand the determinants of undernutrition. The literature from different parts of the world suggest the relationship between malnutrition and dietary habit, environmental, cultural, socioeconomic and demographic factors. ${ }^{6}$ Until the 1980s, the risk factors of undernutrition were limited to dietary habit, agricultural, income, power of purchase, salary and the capacity of the child to intake nutrients. ${ }^{7}$ It was only in the early 1990s that childcare was recognised as one of the major determinants of undernutrition along with food safety, environmental health and access to health services. ${ }^{8}$

Diminished maternal mental health, prepartum and postpartum depression in particular, are major risk factors for underdevelopment of infant and young children compared with infant and young children whose mothers are not depressed. ${ }^{9-11}$ This can have an impact on the mothers' capacity of child care which ultimately increases the risk of infection, malnutrition, impaired child growth and behavioural problems that might be present in adulthood too. ${ }^{911}$ Furthermore, psychological disturbances as depression and anxiety during pregnancy are associated with poor antenatal care, low-birth weight and preterm delivery, where as in postpartum, it is linked with reduced emotional involvement, neglect and hostility resulting in compromised care of the new-born. ${ }^{11}$

The global figure presented by WHO (2018) estimates that nearly $10 \%$ of pregnant women and $13 \%$ of postnatal women experience a mental disorder, most commonly depression. ${ }^{12}$ However, it is concentrated more in LMICs where $15.6 \%$ of women suffer from mental health conditions during pregnancy and $19.8 \%$ after birth. ${ }^{12}$ In LMICs, depression and anxiety are two to three times higher during pregnancy and in postnatal period than in women in high-income countries (HICs) ${ }^{13}$ It is anticipated that the susceptibility to develop mental health problems is probably higher in mothers in LMICs due to low social and economic status, diminished social roles, unwanted pregnancies, poor antenatal care, gender violence and bias. ${ }^{14-16}$ Other risk factors identified were the cultural environment, family size, educational status, difficulties in marriage, immigration and societal displacement, difficult relationship with their mother-in-law and parents, adverse life events during pregnancy and lack of support, woman's relationship with her husband, which is mainly observed in polygamy and alcoholism of husband. ${ }^{15-17}$ Similarly, depression during pregnancy, multi-parity and smoking were also identified to be the risk factors of maternal depression. ${ }^{14-1618-20}$

\section{Why is it important to do this review?}

Globally, mothers and their social surroundings have varied experience regarding conception and childbirth. ${ }^{21}$ Irrespective of where women live, they are at the risk of developing mental health disorder during pregnancy and after birth, but factors such as poverty, migration, stress, exposure to violence, conflicts, natural disaster and low social support increases the likelihood of mental disorders. ${ }^{12}$

There is some evidence that suggests poor mental health, maternal depression in particular is associated with poor child growth. ${ }^{22-28}$ A study conducted in India and Pakistan found the odds of malnourished children was 2.3, 7.4 and 3.9 times higher, respectively, than children with a normal nutritional status of having a depressed mother. ${ }^{25}$

Child growth is the key indicator of child health and nutritional status. Diminished growth in childhood has an irreversible effect on physical growth, educational performance, economic productivity, work capacity and increasing susceptibility to diseases. ${ }^{29}$ Rapid physical and nearly $80 \%$ of cognitive development occurs during the first 2 years of human life. ${ }^{30}{ }^{31}$ The first 2 years of human life, from conception to the second year which is roughly 1000 days is a crucial foundation for both physical and cognitive development of the child. Children in LMICs due to poverty, poor health services and undernutrition often do not reach the full potential of physical and mental development making them more vulnerable to illness and earlier mortality. ${ }^{31}$ Moreover, the primary caregiver is the sole care provider for the social and nutritional needs of the child in these settings, which makes the child more vulnerable to the effects of mental status of their caregiver as they are entirely dependent on them. ${ }^{32}$ Hence, making the children from LMICs more susceptible to ill health.

Recent studies have shown that there is an association between maternal mental health and undernutrition in children. ${ }^{1022-29} 33{ }^{34}$ However, to the best of our knowledge, the reviews conducted until focus on LMICs and maternal depression only. ${ }^{29} 33$ 35-37 Therefore, this study intends to provide a global perspective on maternal mental health issues and child undernutrition (under 5 children) and if the association between maternal mental health and undernutrition in children has yielded different or similar result. Furthermore, it intends to explore the prevalence and the risk factors associated with copresence of maternal mental health and malnutrition in children across the world.

\section{Aim of the review}

The paper aims to systematically review the studies focusing on association between maternal mental health and child undernutrition and critically assess the findings. For which, the review will use following review questions:

- Does the evidence globally support a likely association between maternal mental health and child undernutrition?

- What are the risk factors associated with copresence of maternal mental health and child undernutrition?

- What is the prevalence of maternal mental health disorder (depressive and anxiety) and undernutrition?

The components of population, exposure, comparator and outcome are as follows: 
- Population: children under 5 years of age and their mothers.

- Exposure: maternal mental health disorders.

- Comparator: no mental disorders in mothers.

- Outcome: undernutrition in children under 5 years of age.

\section{METHODS AND DESIGN}

\section{Participants/populations}

The study population will be the children under 5 years of age (0-5) and their mothers.

\section{Exposures}

The studies reporting on maternal mental health data such as common mental disorders which refers to two broad categories: depressive and anxiety disorders. ${ }^{38}$ Studies that include the validated screening tools such as SRQ-20, EPDS, PHQ-9, Generalised Anxiety Disorder (GAD-2), MINI and MINI plus, Kessler Psychological Distress scale (K10) and General Health Questionnaire used to assess the mental health status of a mother will be used in this review. The study will also include any other validated tools that has been used to screen mental health of mothers. As the review progresses, we will critique the studies included in our review as to whether culturally validated tools were used. We will consider the types of tools used while synthesising the findings from different studies. However, we do realise that all the screening tools may not be standardised and valid across different cultures and settings. These will be addressed as limitations for the study.

\section{Study design}

This review will include observational studies that explore the association between maternal mental health and child nutrition. These include prospective, crosssectional, longitudinal, case-control and cohort studies. In addition, the interventional studies such as community trials with the outcome that shows the association between maternal mental health and child nutritional status will also be included in the study. Papers that report the results of association between maternal mental health and child's nutritional status will only be included. The studies that used WHO standard measurement based on weight for height assessment, height for age, stunting or wasting or mid upper arm to assess nutritional status of the child will be included in the review.

\section{Controls}

The control groups will be the mothers without any mental health disorders.

\section{Outcomes}

\section{Primary outcome}

The primary outcome of interest is to assess the association of maternal mental health and child undernutrition. The study will explore association of maternal mental health with all forms of acute and chronic undernutrition.

\section{Secondary outcome}

The study will also analyse mediating factors or risk factors associated with copresence of maternal mental health and child undernutrition. Other important outcomes include the prevalence of maternal mental health disorder and under nutrition in children, age under 5 .

\section{Study period}

The study screening and data extraction process will be completed by February 2021. The final analysis and writing is expected to finish by April 2021.

\section{Patient and public involvement}

As this review will conduct a secondary data analysis from the already available information, it requires no patient involvement.

\section{Search strategy}

The literature search will start with two key words search 'maternal mental health' and 'child malnutrition'. A subheading search will be conducted to identify important studies and ensure literature saturation (see table 2). Furthermore, bibliographies of those papers that match the eligibility criteria will be searched to identify any further relevant article. Two researchers will screen references for eligibility independently. The studies that require consultation with experts in the relevant field and reference checking will be done. The electronic searches will be conducted from following electronic database: MEDLINE (PubMed), PsycINFO, CINAHL, Cochrane Library, Global Health Library Relevant reports from the WHO, United Nations of Children Education Fund (UNICEF) and other organisations working in maternal and child health will also be assessed. Database of systematic reviews (CDSR) and database of abstracts of reviews of effects (DARE) will also be searched for relevant literature (table 1). However, the literature available in English will only be included in the study. The search of literature will be limited from 1995 to 2020 . The study period is limited to the past 25 years

\begin{tabular}{ll}
\hline Table 1 & Search methods \\
\hline $\begin{array}{l}\text { Electronic } \\
\text { searches }\end{array}$ & MEDLINE (PubMed) \\
& PsychINFO \\
& CINAHAL \\
& Cochrane Library \\
& Global Health Library Relevant reports from: \\
& WHO \\
& CDSR/DARE database \\
& United Nations of Children Education Fund \\
& (UNICEF) and other organisation will also be \\
& assessed \\
\hline
\end{tabular}

CDSR, database of systematic reviews; DARE, database of abstracts of reviews of effects; UNICEF, United Nations of Children Education Fund. 


\begin{tabular}{ll}
\hline Table 2 & Search strategy \\
\hline SN & Search term for child malnutrition \\
A1 & malnutrition OR undernutrition OR \\
& inadequate \\
& nutrition OR nutrition disorders OR Stunting \\
& OR \\
& wasting OR Protein energy malnutrition \\
& Search term for maternal mental health \\
& Depression OR anxiety OR psychosis OR \\
& mental \\
& disorder OR Perinatal mental health OR \\
& Postnatal mental health OR Prenatal/ \\
& prepartum Depression OR Postnatal/ \\
& Postpartum Depression
\end{tabular}

\begin{tabular}{ll} 
SN & $\begin{array}{l}\text { Search } \\
\text { S1 }\end{array}$ \\
S2 & $\begin{array}{l}\text { S2 S7 AND A2 } \\
\text { OR } \\
\text { electronic resources OR reviews OR } \\
\text { conference }\end{array}$ \\
& $\begin{array}{l}\text { materials OR thesis/dissertations } \\
\text { S2 AND studies written in English }\end{array}$ \\
\hline $\begin{array}{l}\text { S3 } \\
\text { Additional } \\
\text { methods for } \\
\text { identifying } \\
\text { relevant } \\
\text { literature }\end{array}$ & $\begin{array}{l}\text { Reference checking and hand searching of } \\
\text { articles }\end{array}$ \\
$\begin{array}{l}\text { Checking } \\
\text { reference and } \\
\text { contacting } \\
\text { experts }\end{array}$ & $\begin{array}{l}\text { Reaching or contacting the experts of the } \\
\text { field }\end{array}$ \\
\hline
\end{tabular}

for the following reasons: (i) it was only in the early 1990s, the concept of child care or caring practices was identified as an underlying cause for malnutrition. ${ }^{8}$ (ii) The biggest summit at the time, 'World Declaration and Plan of Action on the Survival, Protection and Development of Children, 1990 ', set seven major goals for implementing declaration, where one of them was 'reducing severe and moderate malnutrition by half of 1990 level among $u-5$ children by the end of the century'. ${ }^{39}$ (iii) This time period captures major milestones in maternal and child health and nutrition including Millennium Development Goals and early years of SDGs. (iv) And finally, we believe time period is sufficient to obtain required data to meet the objective of our systematic review.

A tabular representation of search strategy employed in the study is presented in tables 1 and 2.

\section{Data collection and analysis}

Data extraction and management

Data will be extracted from the relevant papers using online supplemental appendix A (adapted from
Cochrane data collection form) and B. Along with this, different descriptive characteristic as participant characteristics and socioeconomic characteristics available in the study will be extracted.

We will pilot the data extraction form to ensure all the required information has been collected. The data will be extracted based on different themes, study and participant characteristics, result and outcome in regard to the review question. We will repeat each step to ensure the completion of data extraction. Furthermore, the lead author will extract the data from all databases while the three coauthors will perform checks periodically to minimise bias, reduce errors and ensure quality. Any discordance will be resolved through discussion and consensus with all coauthors.

\section{Dealing with duplicate data}

We will use the most completed data set and if feasible, we will collate all available data to generate maximum information.

\section{Risk of bias (quality) assessment}

Assessment of risk bias in included studies

We will use the Cochrane Collaboration's tool for assessment of risk of bias to assess the risk of bias.

\section{Dealing with missing data}

We will obtain any missing data by contacting the authors, when and if feasible.

\section{Assessment of heterogeneity}

The methodological or statistical heterogeneity of the studies will be identified using forest plots and a standard $\chi^{2}$ test. Furthermore, heterogeneity will be assessed using the $\mathrm{I}^{2}$ statistic that quantifies inconsistency across the studies. $^{4041}$

\section{Assessment of reporting bias}

The reporting bias will be assessed using funnel plots. For the possible funnel plots asymmetry, the results will be interpreted carefully.

\section{Data synthesis and analysis of subgroups or subsets Data collection and analysis \\ Selection of studies}

Title, abstract or both will be screened based on the developed search strategy (table 2) to determine which studies should be assessed further. All the potentially relevant articles will be further assessed as full text. To avoid any discrepancies, a review author will ensure if the identified articles meet the inclusion criteria. We will present an adapted Preferred Reporting Items for Systematic Reviews and Meta-Analyses) flowchart for the study selection.

\section{Data synthesis}

After the assessment of methodological heterogeneity, if studies are considered similar enough, then statistical heterogeneity will be looked, and meta-analysis will be conducted if applicable for data synthesis. If the variability 
is too high across the studies, narrative synthesis will be conducted instead.

Either of the analysis that is feasible will be conducted to summarise, show association between study outcomes and explain the factors associated with maternal mental health and child nutritional status. Both textual synthesis and tabular synthesis of data will be provided. An overreaching framework for the synthesis process will be developed where preliminary synthesis, exploring relationships and assessing synthesis robustness will be conducted.

\section{Subgroup analysis and investigation of heterogeneity}

We will carry out the following subgroup analysis and plan to explore the interaction accordingly:

1. Age group: the analysis will be conducted on the following age group of children. Age group 0-2 years or the 1000-day period is chosen as a category as it is widely known as the window of opportunity wherein significant physical and mental development occurs. ${ }^{30} 31$ Children from 2 to 5 years formed the other category.

- (0-2) years.

- (2-5) years.

2. Sex: the analysis of association of maternal mental health and child undernutrition will also be investigated in relation to the sex of the children.

3. We intend to disaggregate the findings if possible, into HIC and LMICs as well.

\section{Sensitivity analysis}

Sensitivity analysis will be performed on the following factors when applicable:

1. Limiting the analysis to the published studies

2. Limiting the analysis by considering the risk of bias, as specified in the section 'Assessment of risk of bias in included studies'.

3. Limiting the analysis of studies to following filters: diagnostic criteria, language of publication, age of participants and study design.

\section{DISCUSSION}

The systematic review protocol will present the global evidence of any association between maternal mental health and undernutrition in children under 5 years of age. The proposed review is likely to be the first review to summarise the potential association between maternal mental health and undernutrition in children at a global level.

A sub-group analysis will be conducted to understand whether the association varies by sex and age group. The analysis performed is expected to provide a comprehensive understanding of the relationship and possibly lead to development of different strategies to address different groups.

\section{ETHICS AND DISSEMINATION}

Ethical approval is not required to conduct this review. The results from the review will be disseminated through peer-reviewed publications, conferences and presentations to policymakers and practitioners where feasible.

Twitter Tomasina Stacey @tomasinastacey

Contributors MS wrote the review protocol, conducted the literature search and wrote the final draft of the manuscript. TS, JA and PS supported MS for articles scanning, data extraction and quality assessment and contributed to the initial drafts. TS, JA and PS reviewed and edited the manuscript. All authors read and approved the final manuscript.

Funding The authors have not declared a specific grant for this research from any funding agency in the public, commercial or not-for-profit sectors.

Competing interests None declared.

Patient consent for publication Not required.

Provenance and peer review Not commissioned; externally peer reviewed.

Supplemental material This content has been supplied by the author(s). It has not been vetted by BMJ Publishing Group Limited (BMJ) and may not have been peer-reviewed. Any opinions or recommendations discussed are solely those of the author(s) and are not endorsed by BMJ. BMJ disclaims all liability and responsibility arising from any reliance placed on the content. Where the content includes any translated material, BMJ does not warrant the accuracy and reliability of the translations (including but not limited to local regulations, clinical guidelines, terminology, drug names and drug dosages), and is not responsible for any error and/or omissions arising from translation and adaptation or otherwise.

Open access This is an open access article distributed in accordance with the Creative Commons Attribution Non Commercial (CC BY-NC 4.0) license, which permits others to distribute, remix, adapt, build upon this work non-commercially, and license their derivative works on different terms, provided the original work is properly cited, appropriate credit is given, any changes made indicated, and the use is non-commercial. See: http://creativecommons.org/licenses/by-nc/4.0/.

\section{ORCID iDs}

Manisha Singh http://orcid.org/0000-0002-4640-8453

Tomasina Stacey http://orcid.org/0000-0003-2002-6200

\section{REFERENCES}

1 World Health Organization. Malnutrition, 2020. Available: https:// www.who.int/health-topics/malnutrition\#tab=tab_1 [Accessed 19 April 2020].

2 WHO. Fact sheets - Malnutrition, 2020. Available: https://www.who. int/news-room/fact-sheets/detail/malnutrition [Accessed 14 Sep 2020].

3 WHO. Children: improving survival and well-being. Available: https:// www.who.int/news-room/fact-sheets/detail/children-reducingmortality [Accessed 18 Sep 2020].

4 Sathyamala C. Global nutrition report: towards a global governance in nutrition. Dev Change 2017;48:1227-42.

5 Pelletier DL, Frongillo EA. Community and international nutrition changes in child survival are strongly associated with changes in malnutrition in developing countries 1,2, 2003. Available: https:// academic.oup.com/jn/article/133/1/107/4687624 [Accessed 8 Feb 2021].

6 Silveira KBR, Alves JFR, Ferreira HS, et al. Association between malnutrition in children living in slums, maternal nutritional status, and environmental factors. J Pediatr 2010;86:215-20.

7 Jonsson U. Hunger and society the causes of hunger hunger as a pressing global problem of human survival, 1981.

8 UNICEF. The state of the World's children. Available: https://www. unicef.org/media/84766/file/SOWC-1998.pdf

9 Satyanarayana VA, Lukose A, Srinivasan K. Maternal mental health in pregnancy and child behavior. Indian J Psychiatry 2011;53:351.

10 Haithar S, Kuria MW, Sheikh A, et al. Maternal depression and child severe acute malnutrition: a case-control study from Kenya. BMC Pediatr 2018;18:289.

11 Pereira PK, Lima LA, Legay LF, et al. Maternal mental disorders in pregnancy and the puerperium and risks to infant health. World $J$ Clin Pediatr 2012;1:20.

12 WHO. Mental health and substance use. Available: https://www.who int/teams/mental-health-and-substance-use/maternal-mental-health [Accessed 19 May 2020].

13 Lund C. Poverty and mental health: towards a research agenda for low and middle-income countries. commentary on Tampubolon and Hanandita (2014). Soc Sci Med 2014;111:134-6. 
14 Prince M, Patel V, Saxena S, et al. No health without mental health. Lancet 2007;370:859-77.

15 Ho-Yen SD, Bondevik GT, Eberhard-Gran M, et al. Factors associated with depressive symptoms among postnatal women in Nepal. Acta Obstet Gynecol Scand 2007;86:291-7.

16 Giri RK, Khatri RB, Mishra SR, et al. Prevalence and factors associated with depressive symptoms among post-partum mothers in Nepal. BMC Res Notes 2015;8:111.

17 Azad R, Fahmi R, Shrestha S, et al. Prevalence and risk factors of postpartum depression within one year after birth in urban slums of Dhaka, Bangladesh. PLoS One 2019;14:e0215735.

18 Chandran M, Tharyan P, Muliyil J, et al. Post-partum depression in a cohort of women from a rural area of Tamil Nadu, India. incidence and risk factors. Br J Psychiatry 2002;181:499-504.

19 Gulamani SS, Premji SS, Kanji Z, et al. Preterm birth a risk factor for postpartum depression in Pakistani women. OJD 2013;02:72-81.

20 Clarke K, Saville N, Shrestha B, et al. Predictors of psychological distress among postnatal mothers in rural Nepal: a cross-sectional community-based study. J Affect Disord 2014;156:76-86.

21 Kumar R. Postnatal mental illness: a transcultural perspective. Springer-Verlag, 1994.

22 Ashaba S, Rukundo GZ, Beinempaka F, et al. Maternal depression and malnutrition in children in Southwest Uganda: a case control study. BMC Public Health 2015;15:1303.

23 Harpham T, Huttly S, De Silva MJ, et al. Maternal mental health and child nutritional status in four developing countries. J Epidemiol Community Health 2005;59:1060-4.

24 Rahman A, Malik A, Sikander S, et al. Cognitive behaviour therapybased intervention by community health workers for mothers with depression and their infants in rural Pakistan: a cluster-randomised controlled trial. Lancet 2008;372:902-9.

25 Rahman A, Iqbal Z, Bunn J, et al. Impact of maternal depression on infant nutritional status and illness: a cohort study. Arch Gen Psychiatry 2004;61:946-52.

26 Nguyen PH, Friedman J, Kak M, et al. Maternal depressive symptoms are negatively associated with child growth and development: evidence from rural India. Matern Child Nutr 2018;14:e12621.

27 Nguyen PH, Saha KK, Ali D, et al. Maternal mental health is associated with child undernutrition and illness in Bangladesh, Vietnam and Ethiopia. Public Health Nutr 2014;17:1318-27.

28 Medhin G, Hanlon C, Dewey M, et al. The effect of maternal common mental disorders on infant undernutrition in Butajira, Ethiopia: the P-MaMiE study. BMC Psychiatry 2010;10:32.
29 Surkan PJ, Kennedy CE, Hurley KM. Depresión materna Y crecimiento durante La primera infancia en Los países en vías de desarrollo: Revisión sistemática Y metaanálisis. Bull World Health Organ 2011;89:607-15.

30 Cusick SE, Georgieff MK. The Role of Nutrition in Brain Development: The Golden Opportunity of the "First 1000 Days". J Pediatr 2016;175:16-21.

31 UNICEF. The first 1,000 days of life: The brain's window of opportunity. Available: https://www.unicef-irc.org/article/958-thefirst-1000-days-of-life-the-brains-window-of-opportunity.htm

32 Black RE, Allen LH, Bhutta ZA, et al. Maternal and child undernutrition: global and regional exposures and health consequences. Lancet 2008;371:243-60.

33 Patel V, Rahman A, Jacob KS, et al. Effect of maternal mental health on infant growth in low income countries: new evidence from South Asia. BMJ 2004;328:820-3.

34 Anato A, Baye K, Tafese Z, et al. Maternal depression is associated with child undernutrition: a cross-sectional study in Ethiopia. Matern Child Nutr 2020;16:e12934.

35 Dadi AF, Miller ER, Mwanri L. Postnatal depression and its association with adverse infant health outcomes in low- and middleincome countries: a systematic review and meta-analysis. BMC Pregnancy Childbirth 2020;20:416.

36 Hurley KM, Surkan PJ, Black MM. Maternal depression and child growth in developing countries: A focus on the postnatal period. In Handbook of growth and growth monitoring in health and disease. Springer New York, 2012: 2023-46.

37 Parsons CE, Young KS, Rochat TJ, et al. Postnatal depression and its effects on child development: a review of evidence from low- and middle-income countries. Br Med Bull 2012;101:57-79.

38 WHO. Depression and other common mental disorders global health estimates, 2017.

39 UN. UN documents gathering a body of global agreements plan of action for implementing the world Declaration on the survival, protection and development of children in the 1990s plan of action for implementing the world Declaration on the survival, protection, 1990.

40 R R. Heterogeneity and subgroup analyses in Cochrane consumers and communication group reviews: planning the analysis at protocol stage, 2016.

41 Higgins JPT, Thompson SG, Deeks JJ, et al. Measuring inconsistency in meta-analyses. BMJ 2003;327:557-60. 